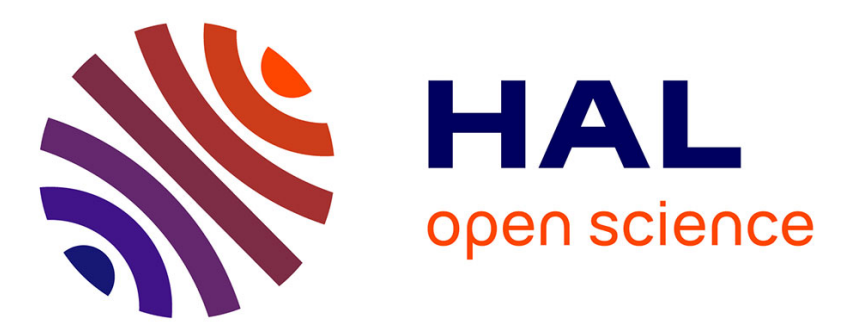

\title{
Effect of the Interfacial Tension on Droplet Association in Aqueous Multiphase Systems
}

\author{
Taco Nicolai, Joaõ P E Machado
}

\section{To cite this version:}

Taco Nicolai, Joaõ P E Machado. Effect of the Interfacial Tension on Droplet Association in Aqueous Multiphase Systems. Langmuir, 2021, 37, pp.5909-5915. 10.1021/acs.langmuir.1c00398 . hal03357470

\author{
HAL Id: hal-03357470 \\ https://hal.science/hal-03357470
}

Submitted on 30 Sep 2021

HAL is a multi-disciplinary open access archive for the deposit and dissemination of scientific research documents, whether they are published or not. The documents may come from teaching and research institutions in France or abroad, or from public or private research centers.
L'archive ouverte pluridisciplinaire HAL, est destinée au dépôt et à la diffusion de documents scientifiques de niveau recherche, publiés ou non, émanant des établissements d'enseignement et de recherche français ou étrangers, des laboratoires publics ou privés. 


\title{
Effect of the Interfacial Tension on Droplet Association in Aqueous Multiphase Systems
}

\author{
Taco Nicolai*a ${ }^{\text {, João P. E. Machado }}{ }^{a, b}$ \\ a'Le Mans Université, IMMM UMR-CNRS 6283, 72085 Le Mans, cedex 9, France \\ ${ }^{\mathrm{b}}$ BioPol, Chemistry Department, Federal University of Paraná, 81.531-980 Curitiba, Paraná, Brazil
}

Email: Taco.Nicolai@univ-lemans.fr

\begin{abstract}
Aqueous multiphase systems (AMPS) were formed by mixtures of three or more incompatible water soluble macromolecules. Droplets formed by different phases in the water-in-water emulsions were found to associate and their morphology was studied using confocal laser scanning microscopy. By analysing the angles between different associated phases it was possible to determine the relative interfacial tensions between phases with respect to each other. In this manner the relative interfacial tension of 15 different pairs of polymers solutions was determined. The effect of the total polymer concentration on the relative interfacial tensions was found to be small as long as mixing of the polymers in the phases was small. The effect of adding protein microgels was studied for systems where they adsorb at the interface between the phases. It is shown that protein microgels can in some cases stabilize associated droplets in suspension.
\end{abstract}

Keywords: Water in water emulsions; polymers; interfacial tension; morphology; phase separation; Pickering emulsion 


\section{Introduction}

When two aqueous solutions of incompatible macromolecules are mixed they tend to phase separate and form an aqueous two phase system (ATPS) also called a water-in-water (W/W) emulsion ${ }^{1-}$

${ }^{3}$. Mixtures of more than two incompatible macromolecules lead to formation of aqueous multiphase systems (AMPS) in which different macromolecules form separate dispersed compartments. AMPS have been studied much less than ATPS, but have attracted attention, because they can be used for selective separation of component that partition between the different compartments ${ }^{4}$. There are also suggestions that AMPS can explain formation of compartments in primitive cells ${ }^{5-6}$.

Droplets containing different macromolecules may interact with each other depending on the relative interfacial tensions. If we consider the case of three phases containing droplets with phases $A$ and $B$ in a continuous phase $C$, the droplets associate if the interfacial tension between phases $A$ and $B\left(\gamma_{A B}\right)$ is smaller than the sum of the interfacial tensions between $A$ and $C$ and between $B$ and $C\left(\gamma_{A B}<\right.$ $\left.\gamma_{A C}+\gamma_{A B}\right)^{7}$. The manner in which the droplets associate depends on the ratios of the interfacial tension. The interfacial tension between two aqueous phase is orders of magnitude lower than that between oil and water and is not easy to determine experimentally ${ }^{8}$. Here we will show how visualization of the association of droplets using confocal laser scanning microscopy (CLSM) can be exploited to determine the interfacial tension even if it is extremely small.

The morphology of emulsions containing two or more distinct dispersed aqueous phases within a continuous aqueous phase has been very little investigated. Song et al. ${ }^{9-10}$ formed water-inwater-in-water emulsions by injected droplets of a dextran/poly(ethylene glycol)(PEG) mixture into a more concentrated PEG solution. Phase separation between dextran and PEG within the droplets was induced by the migration of water from the droplets to the continuous phase, which led to the formation of $A / B / A$ emulsions, with a layer of the dextran phase that surrounded a central PEG phase. A different method to produce $A / B / A$ emulsions not involving microfluidics exploited the temperature dependence of the solubility of maltodextrin in gelatin ${ }^{11}$. W/W emulsions were prepared by mixing gelatin and maltodextrin at high temperatures. Upon decreasing the temperature, the maltodextrin within the gelatin-rich phase that formed the droplets, phase-separated forming small droplets within the gelatin droplets. In both cases the same inner and outer phase was separated by a layer of the second phase. Very recently, a similar method was used in our research group to induced phase separation of gelatin within W/W emulsions formed by amylopectin and guar ${ }^{12}$. In this case the gelatin phase gelled when it cooled. As far as we are aware, the morphology of systems containing two or 
more distinct dispersed liquid phases different from the continuous phase has not yet been investigated.

It has been shown that particles or polymers can in some cases be used to stabilize $\mathrm{W} / \mathrm{W}$ emulsions by forming a layer at the interface that inhibits coalescence between the dispersed droplets 13-14. The driving force for the formation of this layer is thought to be the reduction of the total interfacial tension as was established earlier for oil/water emulsions ${ }^{15}$, but the effect of particles on the interfacial tension has not yet been determined in a systematic manner. Here we have used protein microgels for which the adsorption of microgels at the interface of binary W/W emulsions has already been studied in some detail ${ }^{16-17}$. We will show how the association of different droplets in AMPS is influenced by formation of a layer of microgels at the interface.

\section{Experimental}

Dextran (DEX), poly(ethylene oxide) (PEO), and cold-water fish gelatin (GEL) were obtained from Sigma-Aldrich and their respective weight-average molecular weights $\left(\mathrm{M}_{\mathrm{w}}\right)$ were $1.4 \times 10^{5}, 2.0 \times$ $10^{5}$, and $4.4 \times 10^{5} \mathrm{~g} / \mathrm{mol}$. Pullulan (PUL) with $\mathrm{M}_{\mathrm{w}}=3.0 \times 10^{5} \mathrm{~g} / \mathrm{mol}$ ) and xyloglucan $(\mathrm{XYL})$ with $\mathrm{M}_{\mathrm{w}}=1.0$ $\times 10^{6} \mathrm{~g} / \mathrm{mol}$ were kindly provided by Hayashibara co. and DSP Gokyo Food \& Chemical co., respectively. Amylopectin (AP) from maize with $\mathrm{M}_{\mathrm{w}}=1.0 \times 10^{8} \mathrm{~g} / \mathrm{mol}$ was purchased from Sigma-Aldrich and was purified before use. AP was solubilized at $5 \mathrm{wt} \%$ in a mixture of dimethyl sulphoxide with water 95:5 $\mathrm{v} / \mathrm{v}$. Then, the mixture was centrifuged, and the supernatant was precipitated by adding 3 volumes of ethanol, followed by rinsing with acetone and diethyl ether. The powder was subsequently dried under vacuum for 2 days. All polymers were solubilized in ultra-pure water (Millipore) under stirring. The PEO and the XYL were centrifuged before use in order to remove large particulates. All solutions were set to $\mathrm{pH} 7.0$ by dropwise addition of $\mathrm{HCl}$ or $\mathrm{NaOH}$ at 0.1 and/or $1 \mathrm{~mol} / \mathrm{L}$. Stock solutions of DEX, GEL, PEO, AP, PUL, and XYL were prepared at $30,20,15,15,10$, and $2 \mathrm{wt} \%$, respectively.

Protein microgels (MG) were prepared following a method already described in the literature ${ }^{18}$. Whey protein isolate was solubilized in water at approximately $10 \mathrm{wt} \%$ and centrifuged. The supernatant was subsequently filtrated through $0.2 \mu \mathrm{m}$ pores, the concentration was adjusted to $4 \mathrm{wt} \%$ and the $\mathrm{pH}$ was set to 5.9 before heating for $15 \mathrm{~h}$ at $80^{\circ} \mathrm{C}$. The microgels were analysed by light scattering and the resulting hydrodynamic radius $\left(R_{h}\right)$, radius of gyration $\left(R_{g}\right)$, and $M_{w}$ were $120 \mathrm{~nm}$, $125 \mathrm{~nm}$, and $3.3 \times 10^{8} \mathrm{~g} / \mathrm{mol}$, respectively. The fraction of proteins converted into microgels was approximately $60 \%$, with the residual proteins forming small strands that have been shown not to adsorb at water/water interfaces ${ }^{17}$. 
Confocal laser scanning microscopy (CLSM) observations were done with a Zeiss LSM800 (Carl

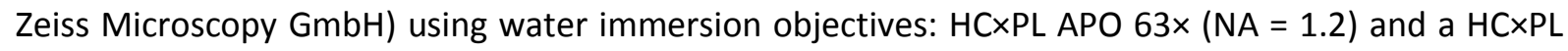
APO $25 \times(N A=0.7)$. The samples were loaded into acrylic wells plates and labelled by adding approximately $5 \mathrm{ppm}$ rhodamine $\mathrm{B}$. The rhodamine physically adsorbed onto the MG, and the excess partitioned differently among the phases, allowing visualization of the different phases. Image J software was used to process the images.

Binary phase diagrams were obtained by mixing two polymers in water at different compositions. Phase separation was checked by observing if two phases could be distinguished in CLSM images. Tie-lines were obtained by measuring the phase volumes after full phase separation induced by centrifugation. In most cases, mixing of the two types of polymers in the separated phases was negligible unless the composition was close to the binodal so that the concentration of polymer in each phase could be calculated from the relative height of the phases.

\section{Results and Discussion}

We have studied AMPS formed by mixing three different types of macromolecules. The mixtures contained volume fractions of approximately $10 \%$ of two phases dispersed in the third continuous phase. For each combination three mixtures were prepared with three different continuous phases. It was found that the relative concentration of macromolecules in each phase was independent of their concentration in the mixture provided that the polymer-polymer miscibility was small. Water distributes between the phases until the equilibrium concentrations are reached, which sets the relative volume fractions of the phases.

As an example, CLSM images for mixtures of amylopectin (AP), poly(ethylene oxide) (PEO) and dextran (DEX) are shown in Fig. 1. The relative concentrations in the phases were found to be approximately $C_{A P}: C_{D E X}: C_{P E O}=4: 2: 1$. The three phases could be visualized simply by adding a small amount of molecular fluorophore rhodamine $B$ that spontaneously partitions differently between the phases. It can be seen that droplets from different phases associate and can form strings of alternating droplets of each phase. 

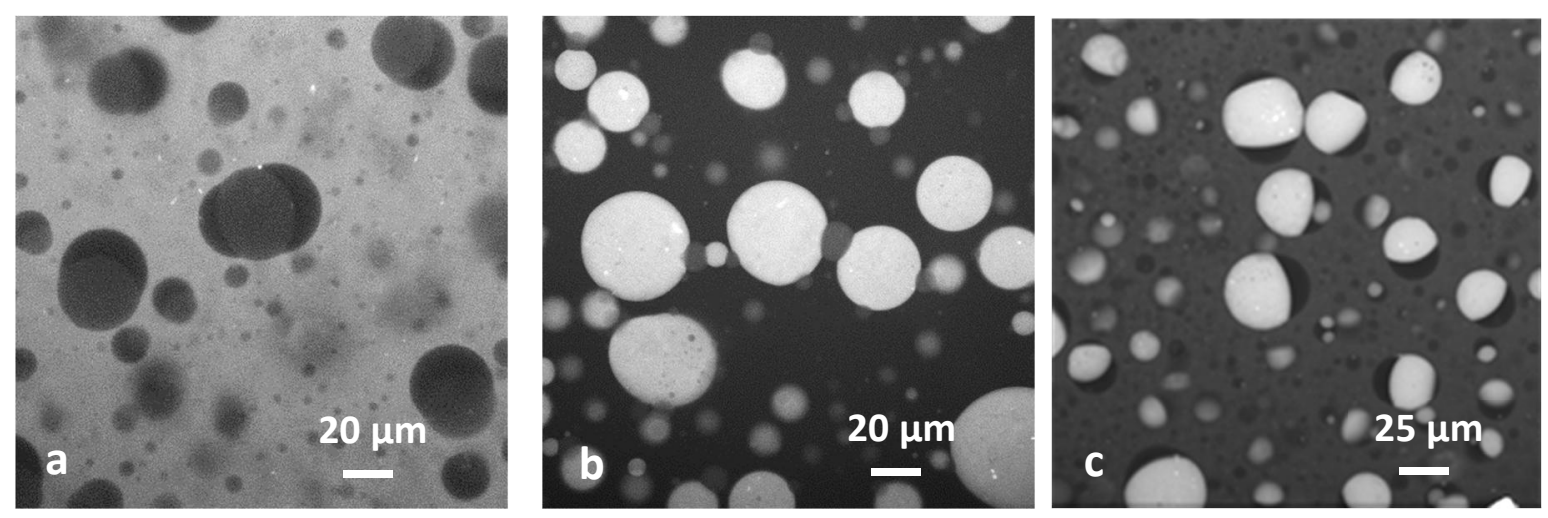

Fig. 1 CLSM images of AMPS containing AP, PEO and DEX with the continuous phase either AP (a), DEX (b) or PEO (c). The brightness increased in the order DEX, PEO, AP caused by the partitioning of the fluorescent marker between the phases.

The angle that each phase forms with the other two phases, see fig. 2 , is determined by the relative interfacial tensions ${ }^{7}$ :

$$
\begin{aligned}
& \cos \left(\theta_{A}\right)=0.5^{*}\left[\left(\gamma_{B C} / \gamma_{A C}\right) \cdot\left(\gamma_{B C} / \gamma_{A B}\right)-\left(\gamma_{A C} / \gamma_{A B}\right)-\left(\gamma_{A B} / \gamma_{A C}\right)\right] \\
& \cos \left(\theta_{B}\right)=0.5^{*}\left[\left(\gamma_{A C} / \gamma_{A B}\right) \cdot\left(\gamma_{A C} / \gamma_{B C}\right)-\left(\gamma_{A B} / \gamma_{B C}\right)-\left(\gamma_{B C} / \gamma_{A B}\right)\right] \\
& \cos \left(\theta_{C}\right)=0.5^{*}\left[\left(\gamma_{A B} / \gamma_{B C}\right) \cdot\left(\gamma_{A B} / \gamma_{A C}\right)-\left(\gamma_{B C} / \gamma_{A C}\right)-\left(\gamma_{A C} / \gamma_{B C}\right)\right\}
\end{aligned}
$$

Inversely, the interfacial tension ratios are related to the angles as follows:

$$
\begin{aligned}
& \gamma_{A B} / \gamma_{B C}=\sin \left(\theta_{C}\right) / \sin \left(\theta_{A}\right) \\
& \gamma_{A B} / \gamma_{A C}=\sin \left(\theta_{C}\right) / \sin \left(\theta_{B}\right) \\
& \gamma_{A C} / \gamma_{B C}=\sin \left(\theta_{B}\right) / \sin \left(\theta_{A}\right)
\end{aligned}
$$

Thus if one of the interfacial tensions is known the other two can be determined by analysing how the two droplets associate. The sum of the three angles is, of course, $360^{\circ}$ and if the interfacial tensions are equal each angle equals $120^{\circ}$. The order of the interfacial tensions can be deduced directly from the order of the angles, eg if $\theta_{A}<\theta_{B}<\theta_{C}$ then $\gamma_{A B}<\gamma_{B C}<\gamma_{A C}$. 


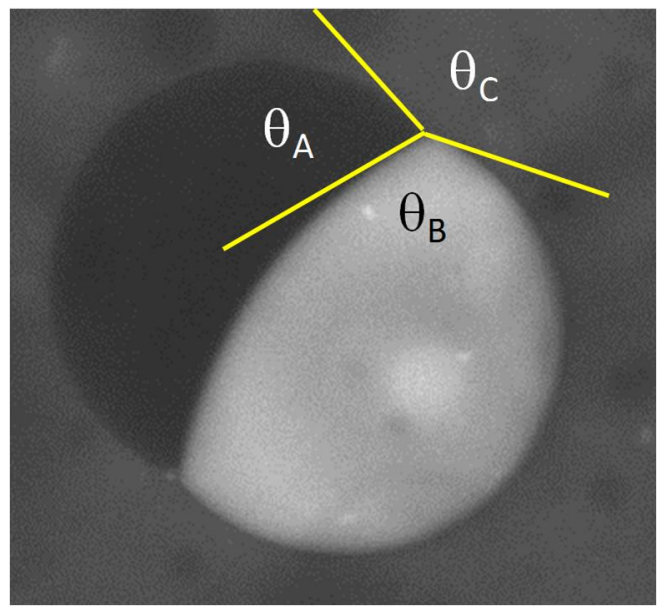

Fig. 2 CLSM image of a DEX droplet (A) associated with a AP droplet (B) in a continuous PEO (C) phase. The angles formed by each phase with the other phases is indicated in the figure.

We found that the angles can be determined more accurately by tracing circles around the two droplets and a third circle that tracks the interface between the two droplets on the images. The angles can be calculated from the radii of the circles and distances between their centres. For more details on the procedure see the supplementary information. Notice that it is important to select images in which the associated droplets are centred in the same horizontal plane or else to take and analyse 3D images. In this manner we found: $\theta_{\mathrm{PEO}}=160^{\circ}, \theta_{\mathrm{AP}}=140^{\circ}$ and $\theta_{\mathrm{DEX}}=70^{\circ}$ for the system shown in Fig. 2 corresponding to $\gamma_{A P / P E O}=1.4 \times \gamma_{D E X / P E O}$ and $\gamma_{A P / D E X}=0.55 \times \gamma_{D E X / P E O}$. Analysing different images of the same system showed that the angles can be determined with an uncertainty of few degrees depending somewhat on the quality of the contrast. The corresponding uncertainty of the relative interfacial tensions depends on the angle itself and becomes large when $\theta$ approaches zero or $180^{\circ} \mathrm{C}$, i.e. when the difference between two interfacial tensions is large.

We investigated the effect of dilution with water for an emulsion of DEX and AP droplets in a continuous PEO phase. The binary phase diagrams of PEO, AP and DEX are shown in Fig. S2 of the supplementary information. As long as the composition was well above the binodals of the AP/PEO, DEX/PEO and AP/DEX binary mixtures, the effect was relatively small with the ratios of the interfacial tensions varying by less than $20 \%$ after diluting by a factor 2.5 , see Fig. S3 of the supplementary information. This is remarkable, because the interfacial tension between DEX and PEO was reported to depend very strongly on the tie-line length (TLL) ${ }^{8,19-21}$. The implication is that the concentration dependences of $\gamma_{D E X / P E O}, \gamma_{A P / P E O}$ and $\gamma_{\text {AP/DEX }}$ are very close. Further dilution had a stronger effect and when the system was diluted by a factor 4.4 , the DEX rich phase almost completely covered the AP rich phase $\left(\theta_{\mathrm{DEX}}=7^{\circ}\right)$, whereas $\theta_{\mathrm{PEO}}$ and $\theta_{\mathrm{AP}}$ were close to $180^{\circ} \mathrm{C}$. The reason is that DEX started to mix 
with PEO ${ }^{22}$ causing a sharp decrease of $\gamma_{\mathrm{DEX} / \mathrm{PEO}}$ with respect to $\gamma_{\mathrm{AP} / \mathrm{PEO}}$ and $\gamma_{\mathrm{AP} / \mathrm{DEX} \text {. After dilution by a }}$ factor 6.6 the PEO and DEX mixed homogeneously, whereas the AP fully mixed with PEO and DEX only after dilution by a factor 15 . Qualitatively similar results were found when other mixtures were diluted.

The relative interfacial tensions can be determined in this manner for any aqueous three phase system as long as none of the interfacial tensions is larger than the sum of the two others. When the latter occurs, the two phases with the highest interfacial tension avoid any contact in the mixtures and the third phase places itself spontaneously in between. Notice that this will always be the case An example is shown in Fig. 3 for mixtures of PEO, DEX and pullulan (PUL). When DEX and PUL are dispersed in PEO, the DEX phase completely surrounds the PUL droplets, and when PEO and DEX are dispersed in PUL, the DEX phase surrounds the PEO droplets. The DEX layer around the PEO phase is not spherical, because the PUL phase was very viscous and the interfacial tension between the DEX and PUL phases ( $\left.\gamma_{\text {PUL/DEX }}\right)$ is very small. In the absence of angles between the phases, the ratio $\gamma_{\text {PUL/DEX }} / \gamma_{\text {PEO/DEX }}$ could not be determined, but it can be deduced that $\gamma_{\text {PUL/DEX }}>\left(\gamma_{\text {PEO/PUL }}-\gamma_{\text {PEO/DEX }}\right)$. Similarly, a layer of DEX was observed to surround PUL droplets dispersed in gelatin (GEL), whereas PUL formed a layer around xyloglucan (XYL) droplets dispersed in PEO. In general, one dispersed phase is expected to surround completely the other dispersed phase if its interfacial tension with one of the other two phases becomes very small, i.e. when the composition of two of the three macromolecules approaches the binodal. To demonstrate this point, the mixture of DEX and AP droplets dispersed in PEO was diluted to very close to the binodal of the DEX/PEO binary system. In that case we observed that the DEX phase completely surrounded the AP droplets, see Fig. S4 of the supplementary information.
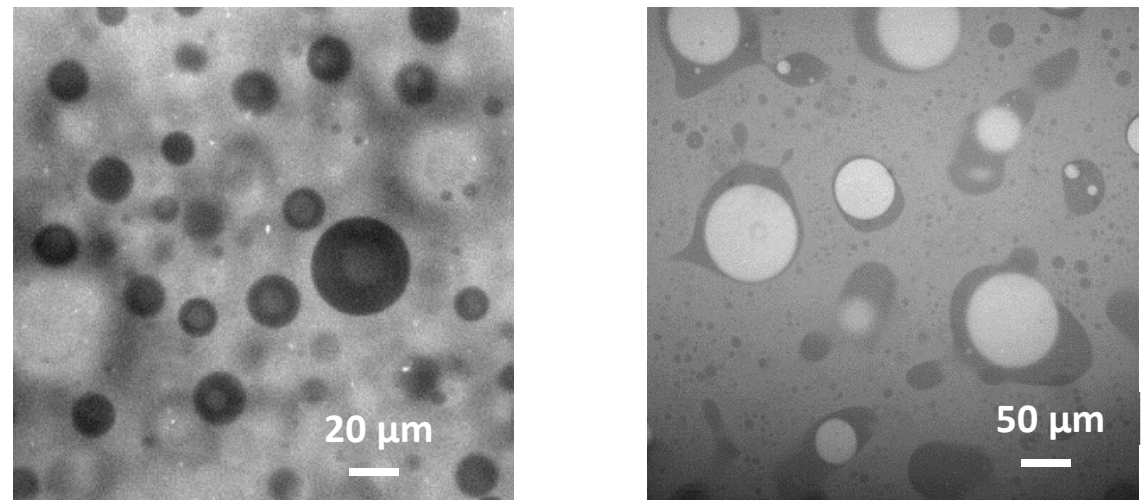

Fig. 3 CLSM images of AMPS formed by DEX and PUL dispersed in PEO (left) and DEX and PEO dispersed in PUL (right). The dextran phase surrounds the dispersed PUL or PEO droplets. 
By analysing CLSM images of different mixtures shown in the supplementary information, the interfacial tensions between a range of different polymers could be compared with each other. Even though $\gamma_{\text {PUL/DEX }}$ could not be compared with $\gamma_{D E X / P E O}$, it could be compared with $\gamma_{A P / D E X}$ and $\gamma_{A P / P U L}$ by analysing mixtures of AP, PEO and PUL. From the relation been $\gamma_{A P / D E X}$ and $\gamma_{P E O / D E X}$ we can deduce the relation between $\gamma_{D E X / P U L}$ and $\gamma_{D E X / P E O}$. In this manner we determined the interfacial tensions for a range of systems with respect to $\gamma_{D E X / P E O}$, see Fig.4. It should be stressed once more that the relative values shown in Fig. 4 are only valid far from the binodal. The maximum concentrations that could be investigated were limited by the viscosity of the continuous phase, but most systems could be studied far above their respective binodals where the relative interfacial tensions depended little on the composition. For most of the systems studied here the interfacial tensions ranged between two times larger and two smaller than $\gamma_{\mathrm{DEX} / \mathrm{PEO}}$. The interfacial tensions between DEX, PUL and XYL were much smaller and depended more strongly on the composition as mixtures of these polymers could not be prepared at compositions far from the binodal. Although the order of the interfacial tension between different systems is relatively robust, the uncertainty in the values varies. As we mentioned above, the uncertainty in the measurement of the angle is a few degrees, but the uncertainty of the relative interfacial tension depends on the angle itself. When one of the angles is closer to $180^{\circ}$, the uncertainty is larger. Therefore, the uncertainty was found to be large when the ratio of interfacial tensions was more than 1.5 or less than 0.5 . From repeated measurements, we found that the uncertainty at intermediate ratios was less than 0.1.

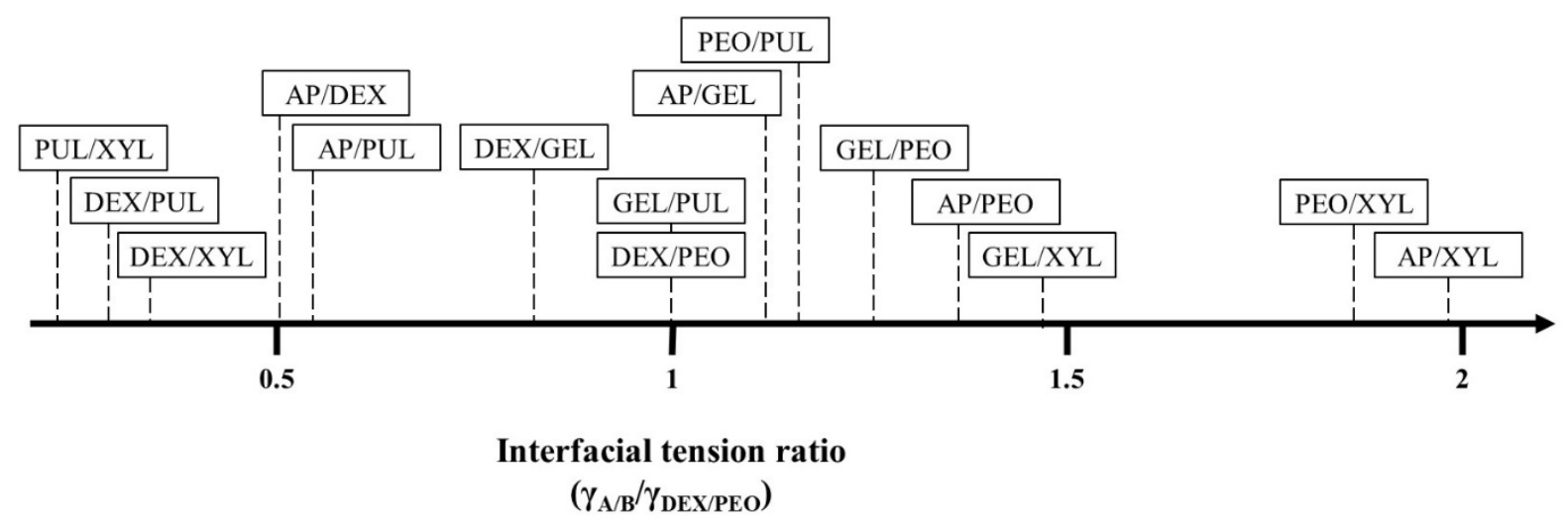

Fig. 4 Comparison of the interfacial tension at interface between the different aqueous polymer solutions studied here with respect to that of the interface between DEX and PEO. 
More complex structures were formed in mixtures of more than three incompatible macromolecules. For example, when DEX, AP and PUL were dispersed in PEO, the PUL and AP phases formed droplets that associated and the DEX phase surrounded the PUL phase shielding it from the PEO phase, see fig. 5a. If in addition GEL was added, the GEL droplets positioned themselves at the PEO/DEX interface, see fig. 5b, in the same manner as in emulsions of GEL and DEX in PEO shown in the supplementary information.
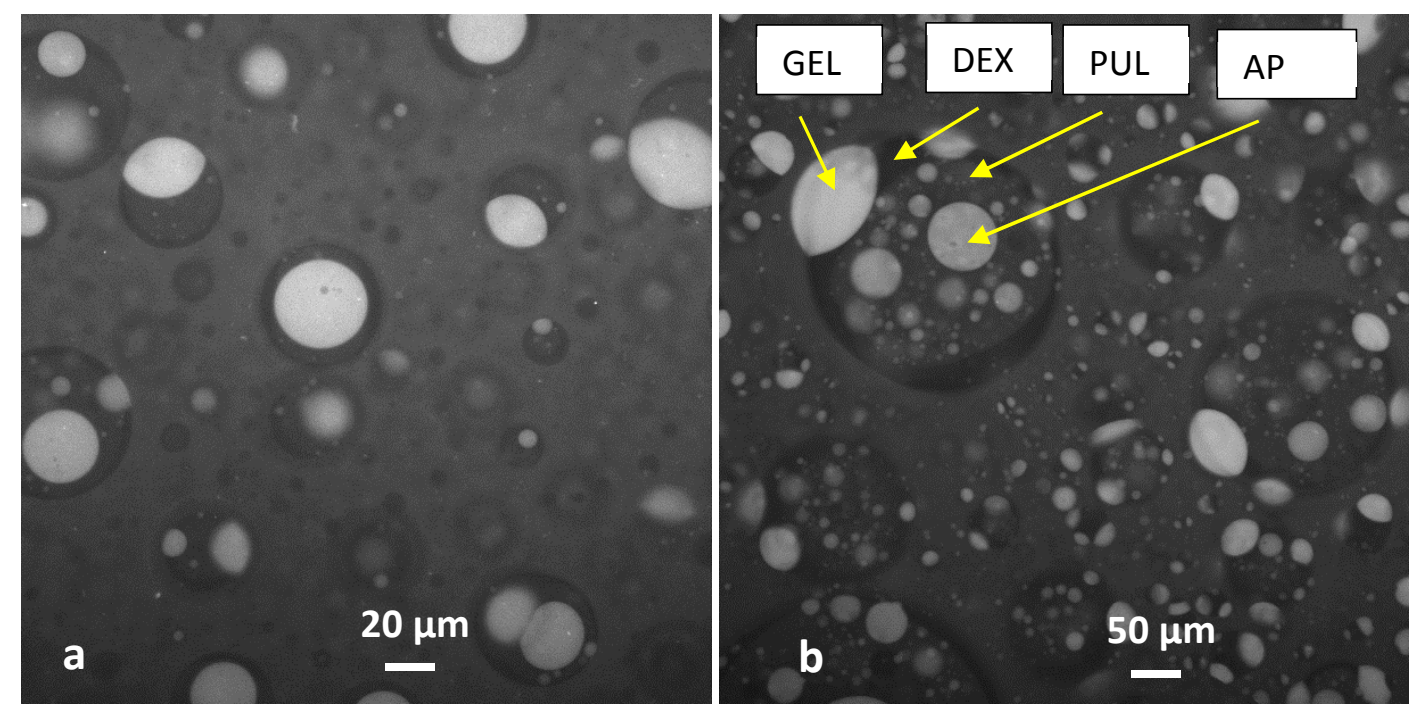

Fig. 5a CLSM images of an AMPS containing AP, PUL and DEX phases dispersed in a continuous PEO phase.

Fig. 5b CLSM images of the same AMPS after adding a small amount of GEL. The different dispersed phases are indicated in the image.

Another example is the AMPS formed by dispersing GEL, AP and PEO in PUL shown in fig. 6a. In this case association of the three types of dispersed droplets led to the formation of strings of droplets. When in addition DEX was added the DEX phase shielded again the PUL phase from the PEO phase, see fig. $6 b$. 


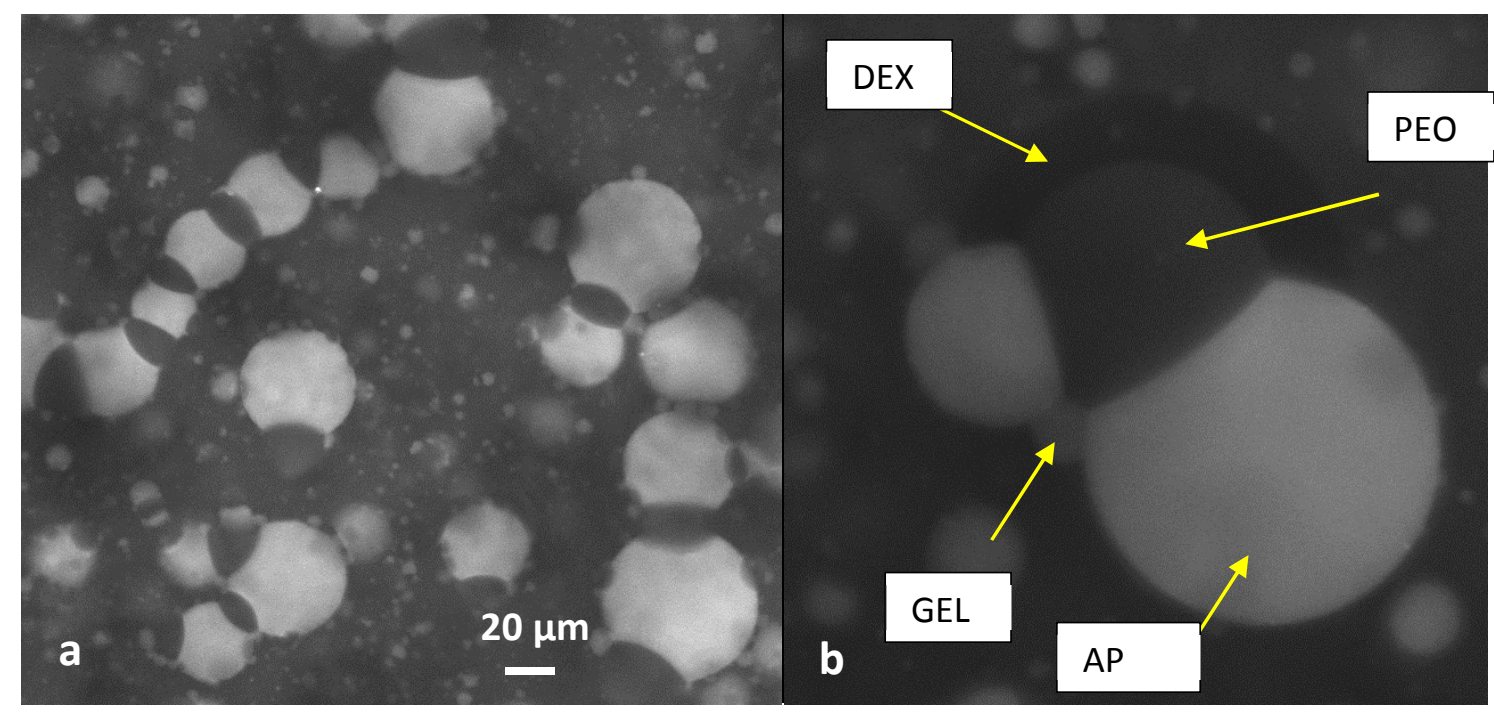

Fig. 6a CLSM image of AP, PEO and GEL phases dispersed in a continuous PUL phase.

Fig. $6 b$ CLSM image of the same AMPS after adding a small amount of DEX. The different dispersed phases are indicated in the image.

\section{Influence of adding microgel particles}

In some sense, one may consider protein microgels as a separate phase that either associates with the dispersed droplets or prefers to place itself within one phase depending on the interfacial tension of the particles with each phase $\left(\gamma_{\mathrm{PA}}, \gamma_{\mathrm{PB}}\right)$. An important difference is, however, that the microgels are solid and therefore have a fixed angle with the other phases. As a consequence, only the angle that the particles make with the interface can vary as a function of the relative interfacial tensions and for spherical particles is given by ${ }^{15}$ :

$\cos \left(\theta_{\mathrm{A}}\right)=1-\cos \left(\theta_{\mathrm{B}}\right)=\left(\gamma_{\mathrm{PB}}-\gamma_{\mathrm{PA}}\right) / \gamma_{\mathrm{AB}}$

Notice that for the same reason the method proposed here cannot be used for mixtures of oil in water. The oil/water interfacial tension is so much larger than the water/water interfacial tension that dispersed oil droplets will remain spherical even if they associate with the aqueous droplets. Particles adsorb at the droplet interface only if $\left|\gamma_{P B}-\gamma_{P A}\right|<\gamma_{A B}$. Elsewhere, it was shown that the microgels adsorb at the PEO/DEX interface ${ }^{17}$ and during this investigation we found that in binary mixtures they also adsorbed at PEO/AP, PEO/PUL and PEO/XYL interfaces. For all other mixtures tested the microgels preferred to stay within one of the phases. The order of preference of the microgels for the systems studied here was found to be from low to high: XYL, AP, PUL, PEO, DEX, GEL. We note that the different preference of the microgels can be exploited to distinguish phases for which the partitioning of rhodamine $B$ is weak, because rhodamine $B$ strongly binds to the microgels. 
Fig. 7 shows AMPS consisting of different combinations of DEX, PUL, and AP droplets dispersed in PEO to which a small amount (0.1-0.2 wt\%) microgels was added. When DEX was present the microgels adsorbed at the DEX/PEO interface. As we have already seen in the absence of microgels, the DEX phase completely surrounds the PUL droplets and associates with the AP droplets. In these emulsions no microgels adsorbed at the other interfaces and excess microgels accumulated preferentially in the DEX phase. The same was observed for DEX/AP/PEO mixtures after dilution to close to the binodal of the DEX/PEO binary system, see Fig S3 of the supplementary information. The situation was different when only AP and PUL droplets were dispersed in PEO. In this case the microgels adsorbed at both the PEO/AP and PEO/PUL interfaces.
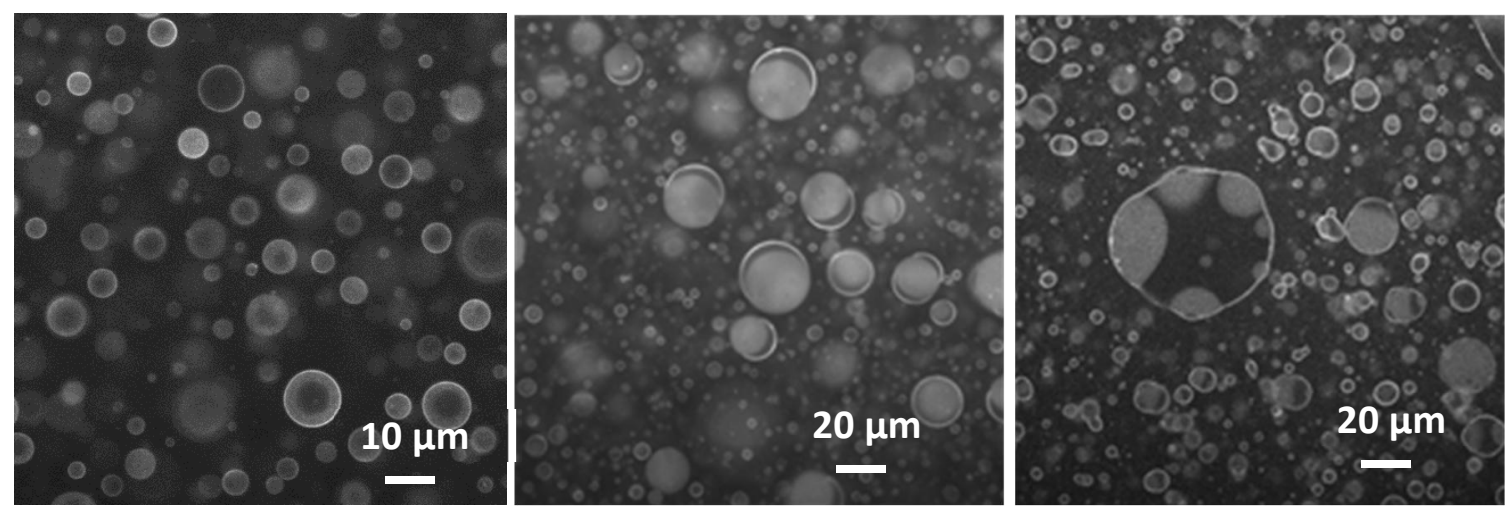

Fig. 7 CLSM images of emulsions formed by DEX and PUL (left) and DEX and AP (middle) and PUL and AP (right) dispersed in PEO containing a small amount of microgels.

Interestingly, the layer of microgels did not have a significant effect on angles between the phases, as is illustrated in fig. 8 . When the microgels cover the interface between phases $A$ and $B, \gamma_{A B}$ is replaced by the weighted sum of the interfacial tensions of the particles with each phase. The effective relative interfacial tensions may therefore be expected to be different causing a change of the angles that the phases make with each other. However, the observation that the angles between the phases were not significantly influenced by the microgels for the systems investigated here implies that the effective interfacial tension was not significantly modified by the presence of the microgels. It should be realized, however, that the microgels at the interface no longer contribute to the free energy of the bulk phases so that even if the effective interfacial tension between the two phases is not reduced the total free energy is lower when the particles adsorb at the interface. Unfortunately, the microgels were too small to be able to determine their contact angle with the interface. It is not clear whether the contact angle of the particles adsorbed at the interface has an influence on the angles 
between the phases. Future measurements with other types of particles having different contact angles may resolve this issue.
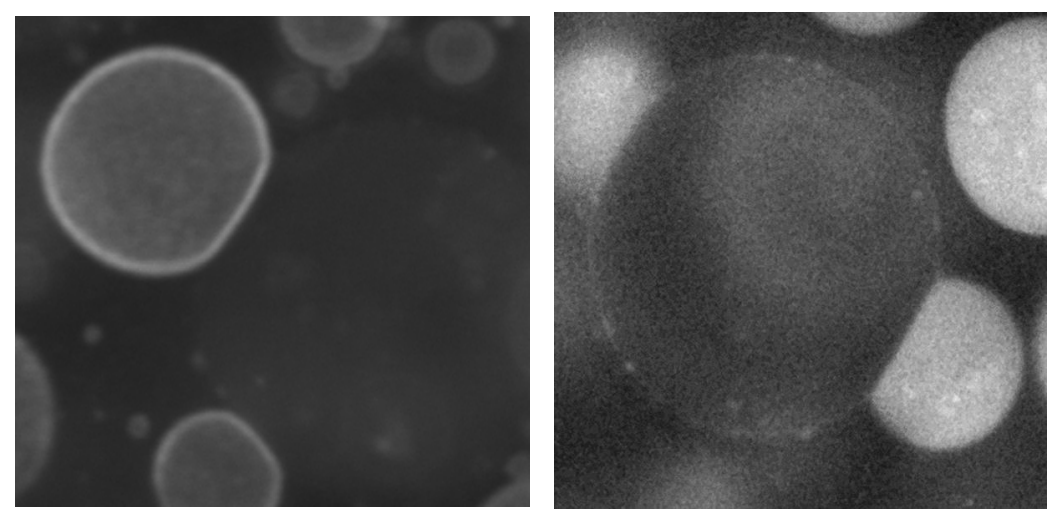

Fig. 8 CLSM images of associated AP and PEO droplets in a continuous PUL phase with (left) and without (right) microgels. The microgels bind strongly the fluorescent marker so that the adsorbed microgels are seen as a bright layer around the AP droplets. Notice that the AP droplets are brightest in the absence of microgels, but that the PEO droplet are brightest in the presence of microgels, because the excess of the latter partition more to the PEO phase.

Formation of a layer of microgels at the interface was reported in the literature to stabilize emulsions of PEO droplets dispersed in DEX against coalescence, though not for DEX droplets dispersed in PEO ${ }^{17}$. In this study we noted that both PUL and AP droplets could be stabilized by microgels when dispersed in PEO. PEO droplets could be stabilized by microgels when dispersed in PUL, but not when dispersed in AP. Interestingly, associated droplets of AP and PUL dispersed in PEO (fig. 7) slowly sedimented, but did not coalescence for at least one week in the presence of microgels, see fig. S6 of the supplementary information. Even more striking was the formation of a stable network formed by association of PEO and AP droplets in a continuous PUL phase, see fig. 9, even though the AP droplets by themselves were not stable. The stable PEO droplets did not associate with each other so that the presence of AP was required to form the network. We note that macroscopically this AMPS behaved as a viscous liquid. These findings show that it is possible to create stable AMPS. 


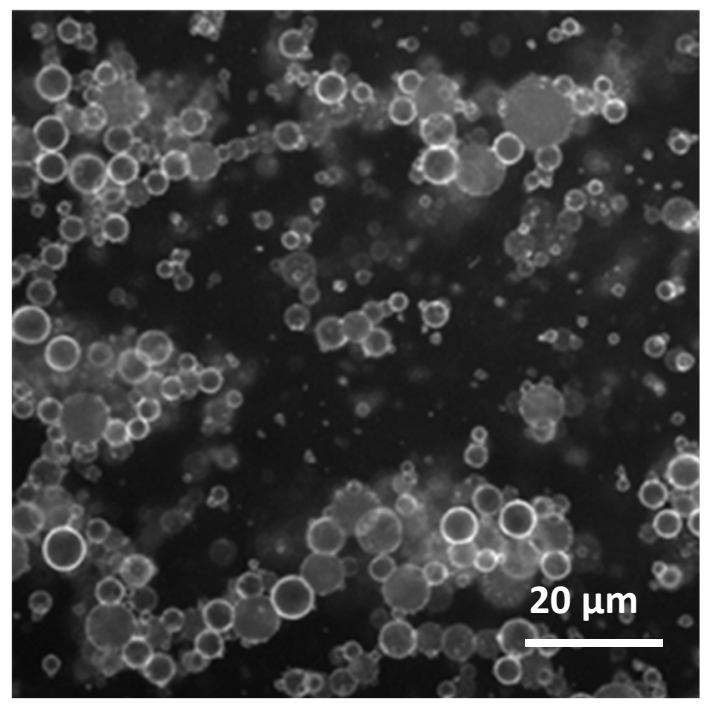

Fig. 9 CLSM images of a network associated AP and PEO droplets in a continuous PUL phase after several days standing. The PEO droplets are less bright than the AP droplets and are surrounded by a layer of microgels.

\section{Conclusions}

The morphology of aqueous multiphase systems is determined by the ratios of the interfacial tensions between the phases. Association between dispersed droplets of two different phases occurs when none of the interfacial tensions is larger than the sum of the two others. In this manner strings can be formed by association of droplets alternating between different phases. If one of the interfacial tensions is higher than the sum of the two others, the two phases with the highest interfacial tension will be entirely separated from each other by the interposing third phase. Measurement of the angles between the phases allows one to calculate the ratio of the interfacial tensions. The ratio depends only weakly on the concentration of the macromolecules in the mixture as long as phase separation is strong, i.e. the systems are far from their respective binodals.

Solid particles can be considered as a distinct phase and either adsorb at the interface between the liquid phases or disperse in one of the phases depending on the interfacial tensions between the particles and the phases with respect to that between the liquid phases. Adsorption of protein microgels at the interface did not significantly modify the way the liquid droplets associated for the systems studied here. Certain AMPS can be stabilized by adding particles in the same manner as binary W/W emulsions. 


\section{ASSOCIATED CONTENT}

Supporting Information. Explanation of the determination of the phase angles. Binary phase diagrams of AP/DEX; DEX/PEO and DEX/AP. CLSM images of associated AP and DEX droplets dispersed in PEO at different dilutions. Images of AP droplets surrounded by the DEX phase dispersed in PEO with and without protein microgels. Images of a range of different AMPS. Images of aged associated PUL and AP droplets dispersed in PEO.

\section{Author information}

\section{ORCID:}

João Pedro Elias Machado: 0000-0003-2820-821X

Taco Nicolai : 0000-0002-7206-1975

\section{Acknowledgement}

This research was financed by the regional programme Food for Tomorrow /Cap Aliment; Research, Education and Innovation in Pays de la Loire, which is supported by the French Region Pays de la Loire and the European Regional Development Fund. The CLSM analyses were conducted within platforms "Matière molle" at IMMM.

\section{References}

1. Frith, W. J., Mixed biopolymer aqueous solutions - phase behaviour and rheology. Adv. Colloid Interface Sci. 2010, 161 (1-2), 48-60.

2. Chao, Y.; Shum, H. C., Emerging aqueous two-phase systems: from fundamentals of interfaces to biomedical applications. Chemical Society Reviews 2020, 49 (1), 114-142.

3. Iqbal, M.; Tao, Y.; Xie, S.; Zhu, Y.; Chen, D.; Wang, X.; Huang, L.; Peng, D.; Sattar, A.; Shabbir, M. A. B., Aqueous two-phase system (ATPS): an overview and advances in its applications. Biological procedures online 2016, 18 (1), 1-18.

4. Akbulut, O.; Mace, C. R.; Martinez, R. V.; Kumar, A. A.; Nie, Z.; Patton, M. R.; Whitesides, G. M., Separation of nanoparticles in aqueous multiphase systems through centrifugation. Nano letters 2012, 12 (8), 4060-4064.

5. Keating, C. D., Aqueous phase separation as a possible route to compartmentalization of biological molecules. Accounts of chemical research 2012, 45 (12), 2114-2124.

6. Mytnyk, S.; Olive, A. G.; Versluis, F.; Poolman, J. M.; Mendes, E.; Eelkema, R.; van Esch, J. H., Compartmentalizing Supramolecular Hydrogels Using Aqueous Multi-phase Systems. Angewandte Chemie 2017, 129 (47), 15119-15123.

7. Rowlinson, J. S.; Widom, B., Molecular theory of capillarity. Courier Corporation: 2013.

8. Ding, P.; Wolf, B.; Frith, W. J.; Clark, A. H.; Norton, I. T.; Pacek, A. W., Interfacial tension in phase-separated gelatin/dextran aqueous mixtures. J Colloid Interface Sci 2002, 253 (2), 367-76.

9. Song, Y.; Sauret, A.; Cheung Shum, H., All-aqueous multiphase microfluidics. Biomicrofluidics 2013, 7 (6), 061301. 
10. Song, Y.; Shum, H. C., Monodisperse w/w/w double emulsion induced by phase separation. Langmuir 2012, 28 (33), 12054-12059.

11. Beldengrün, Y.; Dallaris, V.; Jaén, C.; Protat, R.; Miras, J.; Calvo, M.; Garcia-Celma, M. J.; Esquena, J., Formation and stabilization of multiple water-in-water-in-water (W/W/W) emulsions. Food Hydrocolloids 2020, 102, 105588.

12. Chen, J.; Guo, J.; Yang, X.; Nicolai, T., Water-in-water-in-water emulsions formed by cooling mixtures of guar, amylopectin and gelatin. Food Hydrocolloids 2021, ASAP.

13. Dickinson, E., Particle-based stabilization of water-in-water emulsions containing mixed biopolymers. Trends in Food Science \& Technology 2018, 83, 31-40.

14. Chao, Y.; Shum, H. C., Emerging aqueous two-phase systems: from fundamentals of interfaces to biomedical applications. Chemical Society Reviews 2020, 49 (1), 114-142.

15. Aveyard, R.; Binks, B. P.; Clint, J. H., Emulsions stabilised solely by colloidal particles. Adv. Colloid Interface Sci. 2003, 100, 503-546.

16. Hazt, B.; Bassani, H. P.; Elias-Machado, J. P.; Buzzo, J. L. A.; Silveira, J. L.; de Freitas, R. A., Effect of $\mathrm{pH}$ and protein particle shape on the stability of amylopectin-xyloglucan water-in-water emulsions. Food Hydrocolloids 2020, 104, 105769.

17. Nguyen, B. T.; Nicolai, T.; Benyahia, L., Stabilization of water-in-water emulsions by addition of protein particles. Langmuir 2013, 29 (34), 10658-64.

18. Phan-Xuan, T.; Durand, D.; Nicolai, T.; Donato, L.; Schmitt, C.; Bovetto, L., On the Crucial Importance of the $\mathrm{pH}$ for the Formation and Self-Stabilization of Protein Microgels and Strands. Langmuir 2011, 27, 15092.

19. Balakrishnan, G.; Nicolai, T.; Benyahia, L.; Durand, D., Particles trapped at the droplet interface in water-in-water emulsions. Langmuir 2012, 28 (14), 5921-6.

20. Forciniti, D.; Hall, C.; Kula, M., Interfacial tension of polyethyleneglycol-dextran-water systems: influence of temperature and polymer molecular weight. Journal of Biotechnology 1990, 16 (3-4), 279296.

21. Ding, P.; Pacek, A.; Frith, W.; Norton, I.; Wolf, B., The effect of temperature and composition on the interfacial tension and rheology of separated phases in gelatin/pullulan mixtures. Food Hydrocolloids 2005, 19 (3), 567-574.

22. Edelman, M. W.; Van Der Linden, E.; Tromp, R. H., Phase separation of aqueous mixtures of poly (ethylene oxide) and dextran. Macromolecules 2003, 36 (20), 7783-7790. 


\title{
Supplementary Information
}

\section{Effect of the Interfacial Tension on Droplet Association in Aqueous Multiphase Systems}

\author{
Taco Nicolai* ${ }^{a}$, João P. E. Machado ${ }^{a, b}$ \\ aLe Mans Université, IMMM UMR-CNRS 6283, 72085 Le Mans, cedex 9, France

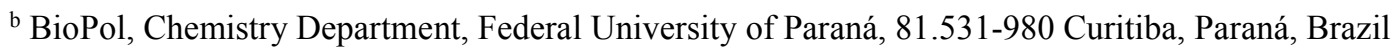

\section{Determination of the angles between phases.}

The angle formed by each phase is related to the angle between circles that trace the different interfaces, see fig S1, which can be calculated from the radii of the circles and the distances between the centres of the circles:

$\cos \left(\theta_{A}\right)=-\left(r_{A}^{2}+r_{c}^{2}-d_{a c}^{2}\right) / 2 r_{A} r_{c}$

$\left.\cos \left(\theta_{B}\right)=\left(r_{b}^{2}+r_{c}^{2}-d_{b c}{ }^{2}\right) / 2 r_{B} r_{c}\right)$

$\cos \left(\theta_{C}\right)=\left(r_{A}^{2}+r_{B}^{2}-d_{a b}^{2}\right) / 2 r_{A} r_{B}$

Here $r_{A}, r_{B}$ and $r_{C}$ are the radii of circles tracing the interfaces $A / C, B / C$, and $A / B$, respectively. $d_{A C}$ represent the distance between the centres of the circles with radii $r_{A}$ and $r_{C}$.

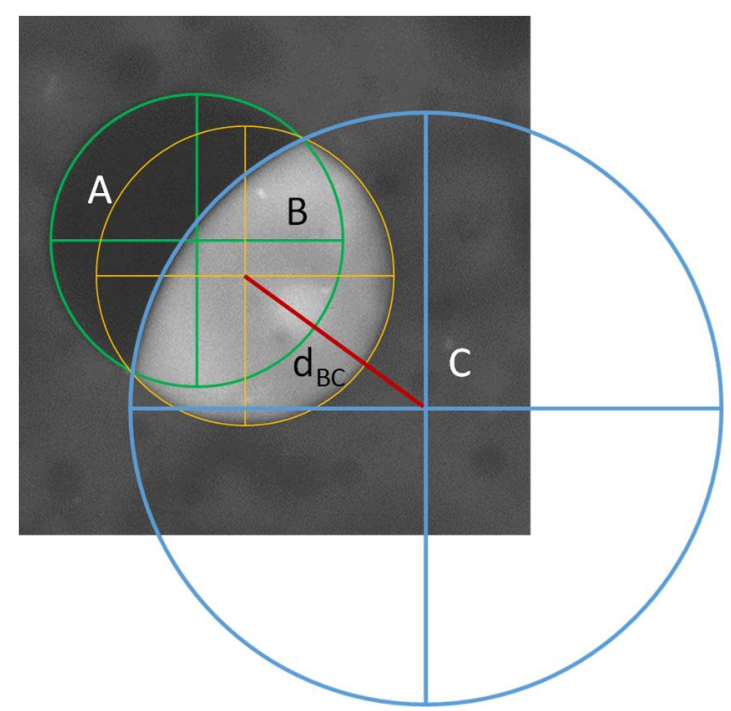

Fig. S1 Example of two associated droplets of phase $A$ and $B$ in continuous phase $C$ with the circles that trace the interfaces. $d_{B C}$ is also indicated. 


\section{Phase diagrams}
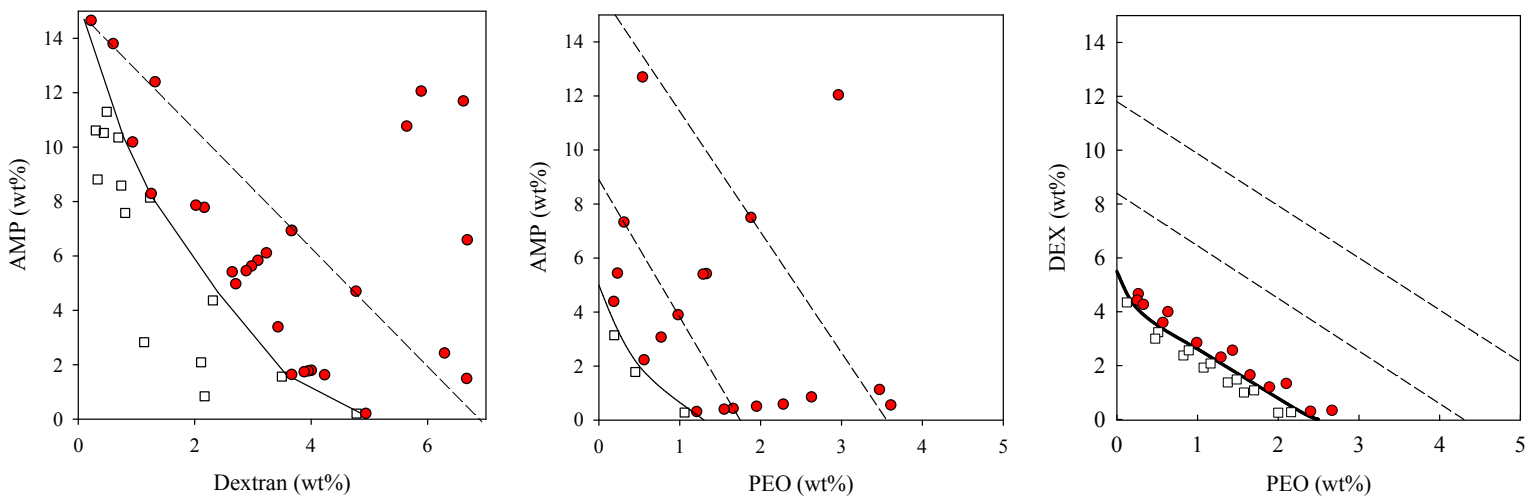

Fig. S2 phase diagrams for binary mixtures of AP, DEX and PEO. The binodals are indicated by solid lines and a few tie-lines are indicated by dashed lines. The phase diagram of the DEX/PEO mixtures was taken from the literature (Gonzalez et al. Langmuir 2016, 32, 7189-7197. 


\section{Effect of dilution}

Fig. S3 shows the effect of dilution with water on the angles between AP DEX and PEO phases. The concentrations in the undiluted system were: 10.5, 2.9 and $3.2 \mathrm{wt} \%$ for PEO, DEX and AP, respectively. The corresponding concentrations in the phases were approximately $13,25,49 \mathrm{wt} \%$ except at stronger dilutions where PEO and DEX begin to mix. At the dilution closest to the binodal of the PEO/DEX binary system, AP droplets completely surrounded by the DEX phase could be observed, see Fig. S4. Added protein microgels formed a layer at the PEO-DEX interface with the excess microgels accumulating in the DEX phase.

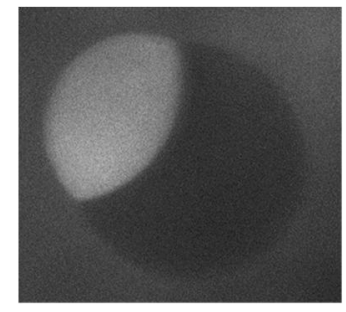

1

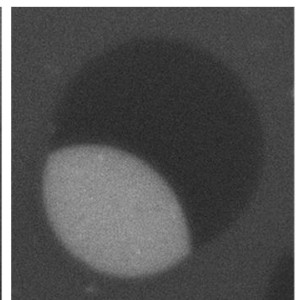

1.5

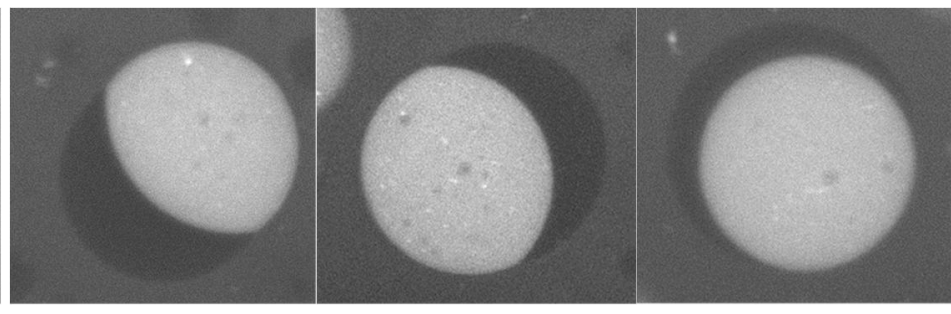

2.5

3.3

4.4

Fig. S3 CLSM images of associated AP (bright) and DEX (dark) droplets in PEO at different dilutions indicated in the figure.
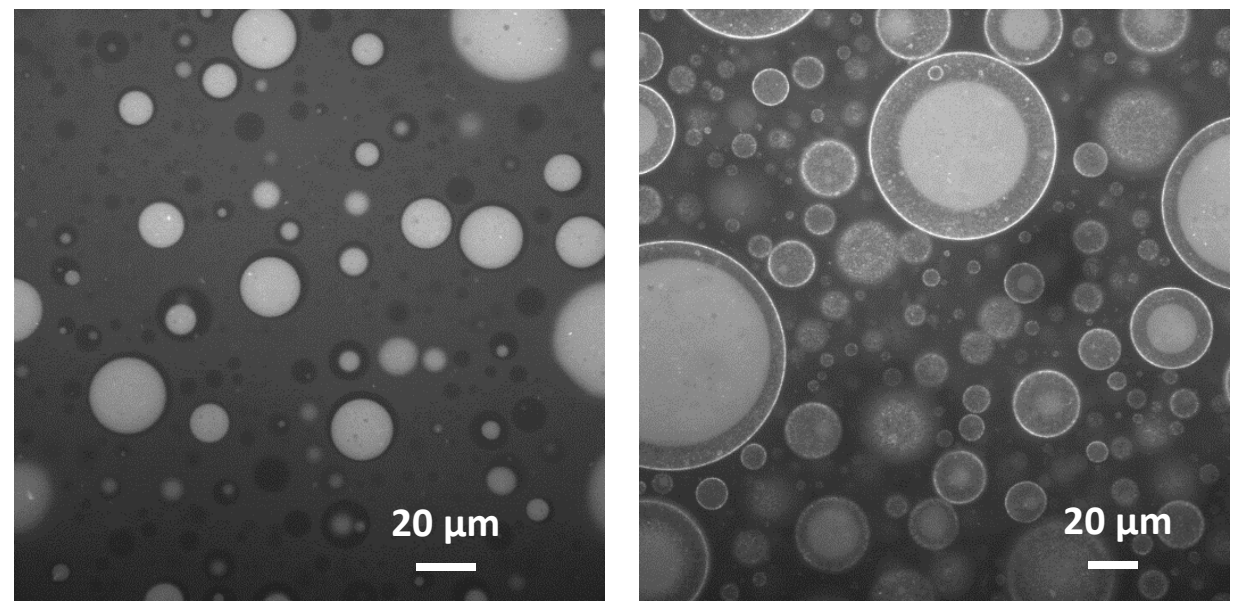

Fig. S4 CLSM images of AP droplets surrounded by the DEX phase dispersed in PEO without (left) and with (right) protein microgels. Notice that the dark DEX layer around the bright AP droplets becomes brighter in the presence of microgels, which strongly bind the fluorescent marker, because the excess microgels partition to the DEX phase. 


\section{CLSM images of different AMPS}

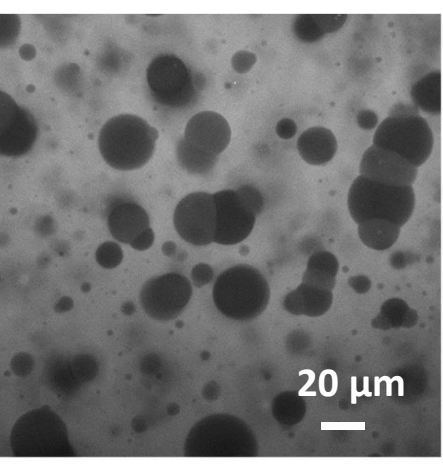

$D E X+P E O$ in GEL

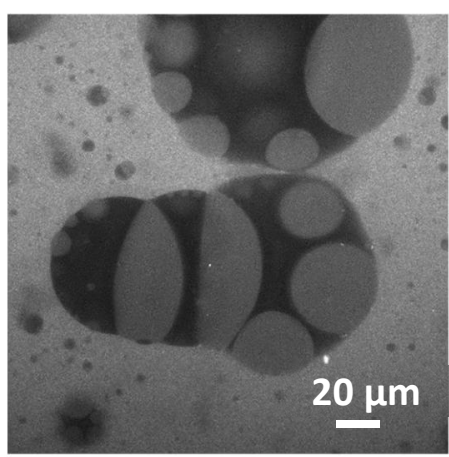

$\mathrm{PUL}+\mathrm{AP}$ in GEL

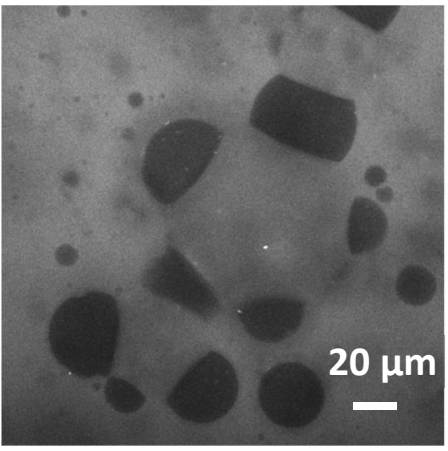

$\mathrm{PEO}+\mathrm{AP}$ in GEL

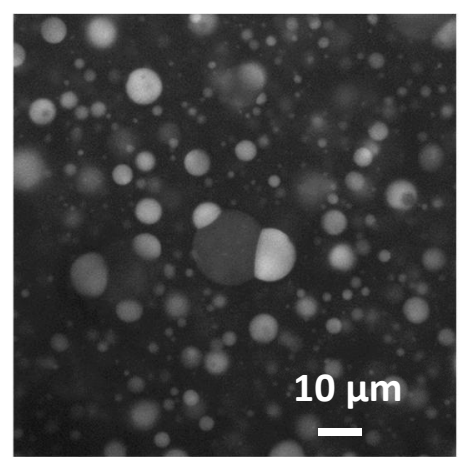

GEL + PEO in DEX

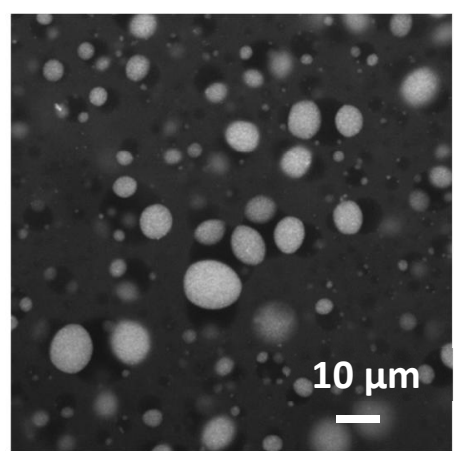

$\mathrm{PUL}+\mathrm{Gel}$ in AP

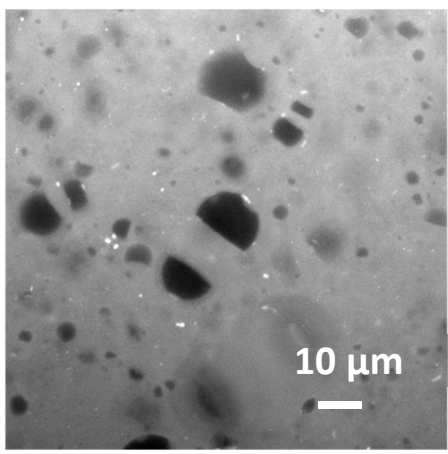

Gel + PEO in AP

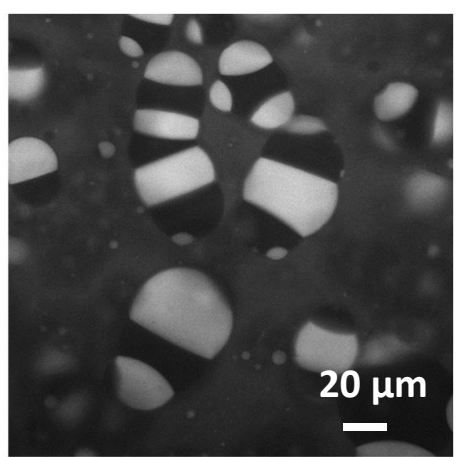

GEL + DEX in PEO

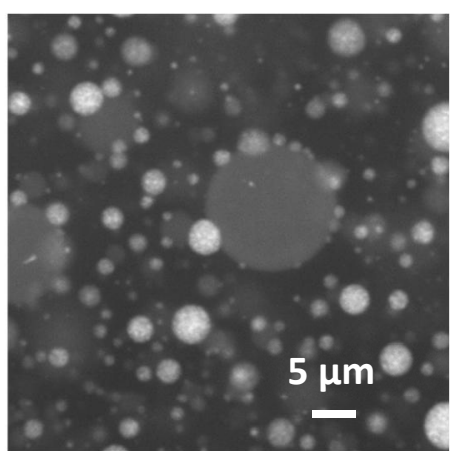

$\mathrm{AP}+\mathrm{Gel}$ in $\mathrm{PUL}$

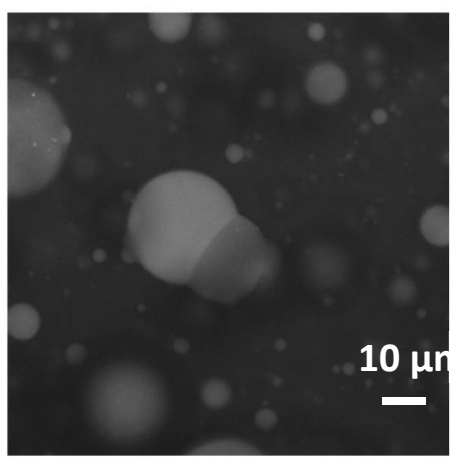

GEL + AP in PEO 


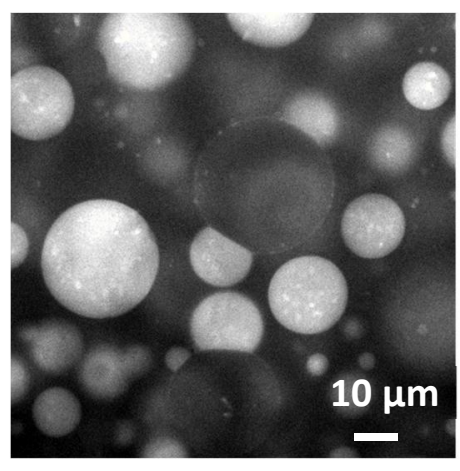

$\mathrm{AP}+\mathrm{PEO}$ in $\mathrm{PUL}$

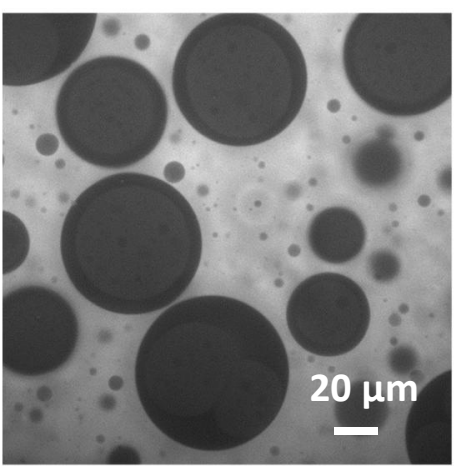

PUL+ DEX in GEL

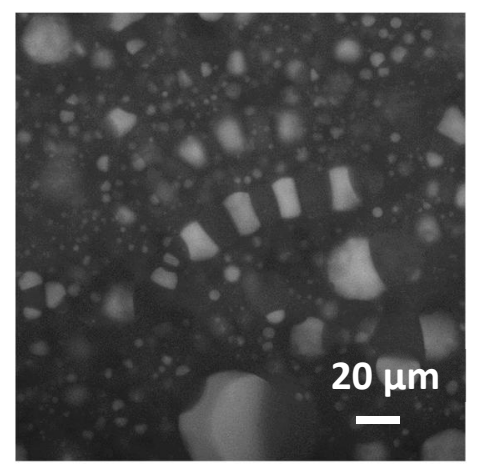

Gel + POE in PUL

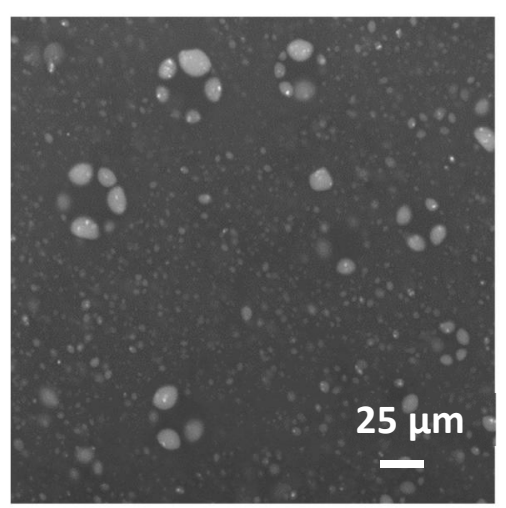

$P E O+A P$ in $X Y L$

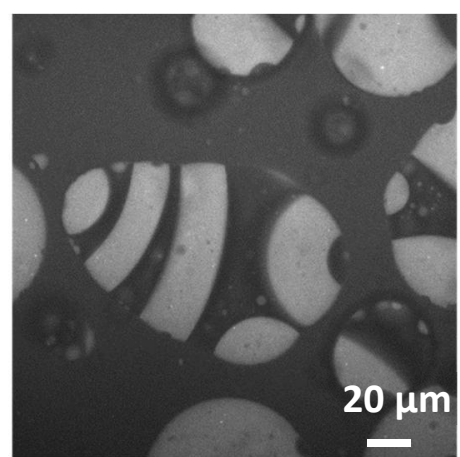

$A P+P U L$ in PEO

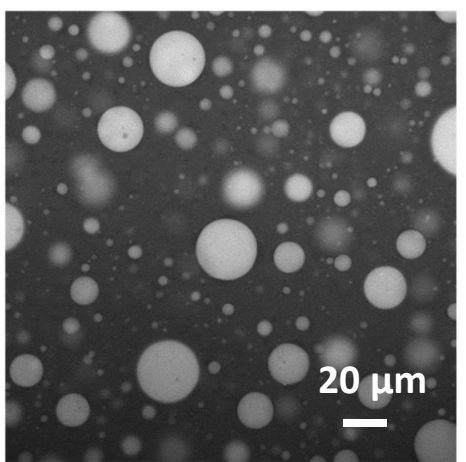

DEX + GEL in PUL

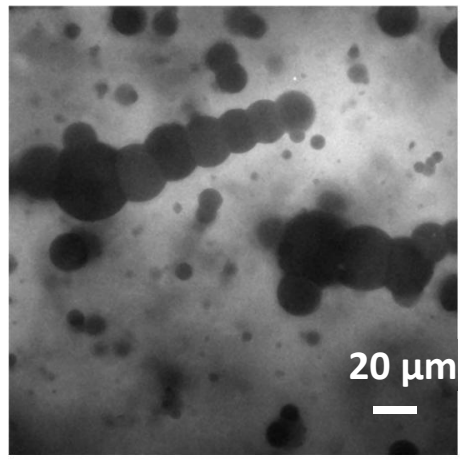

PUL+ PEO in GEL

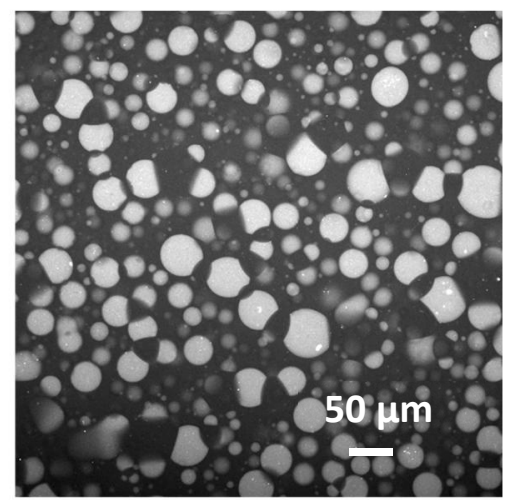

$X Y L+A P$ in $P E O$

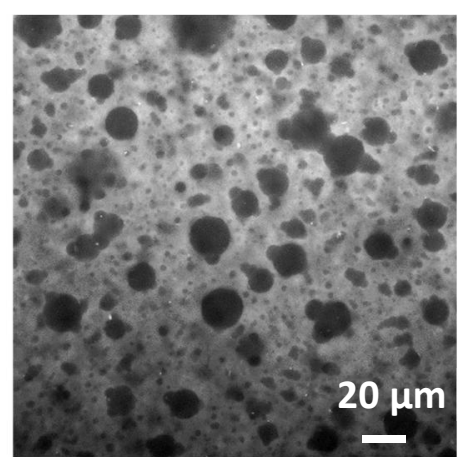

$\mathrm{POE}+\mathrm{PUL}$ in $\mathrm{AP}$

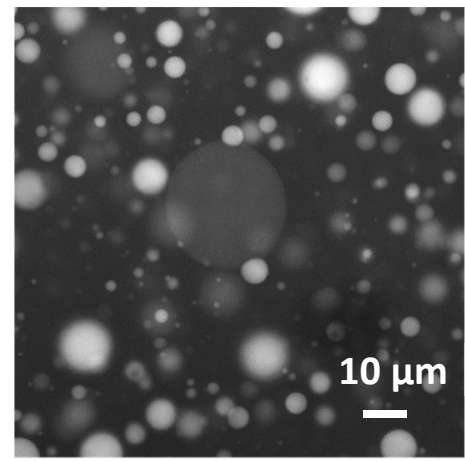

GEL + PUL in DEX

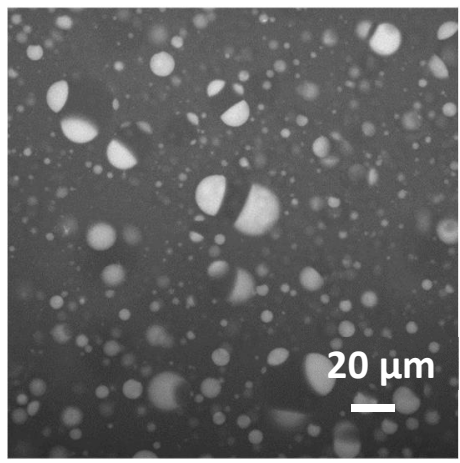

GEL + PUL in PEO 


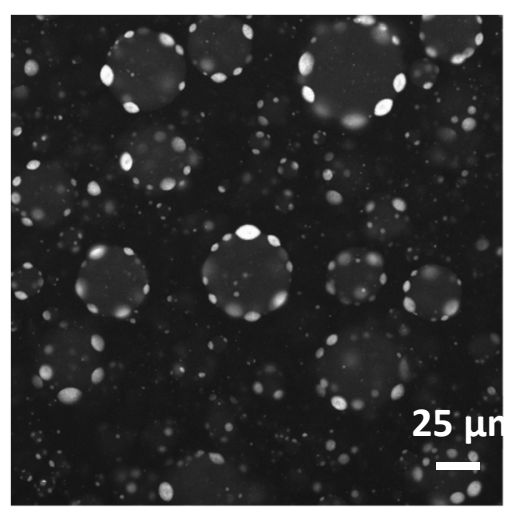

GEL+ AP in XYL

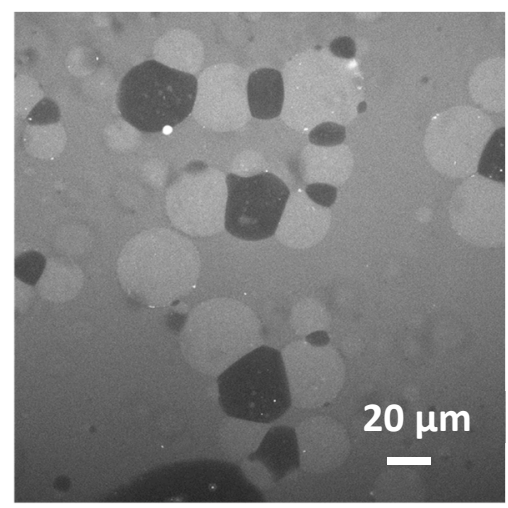

$X Y L+A P$ in $G E L$

Fig. S5 CLSM images of different AMPS as indicated in the figure. The brightness of each phase depends on the partitioning of rhodamin $B$ between the phases. The order with decreasing brightness is: AP, GEL, PEO, PUL, XYL. In the system GEL + AP in XYL microgels were added to enhance the brightness of the GEL phase so that it became brighter than the AP phase. Notice that the scale varies between images.

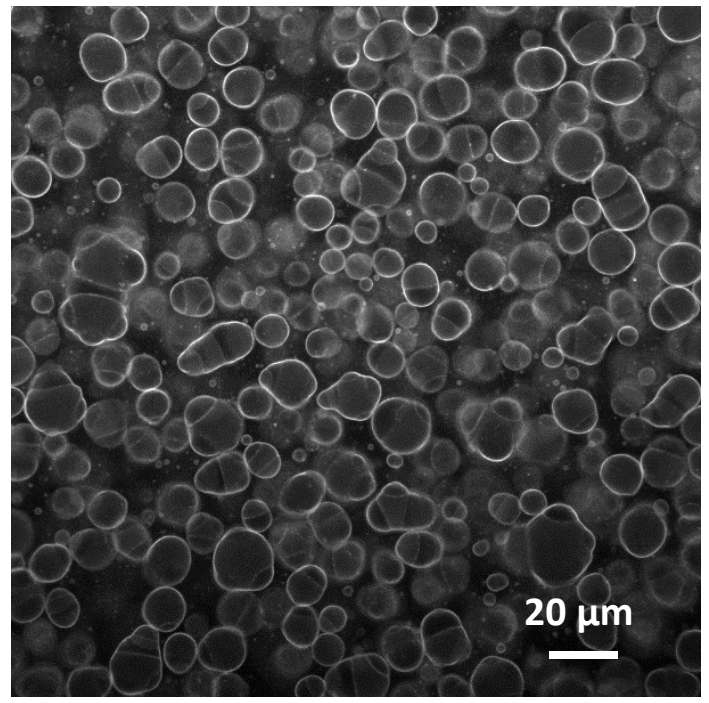

Fig. S6 CLSM image of an AMPS containing associated PUL and AP droplets dispersed in PEO with microgels adsorbed at the interfaces with PEO. The image was taken after one week standing near the bottom and show the sedimented layer. 